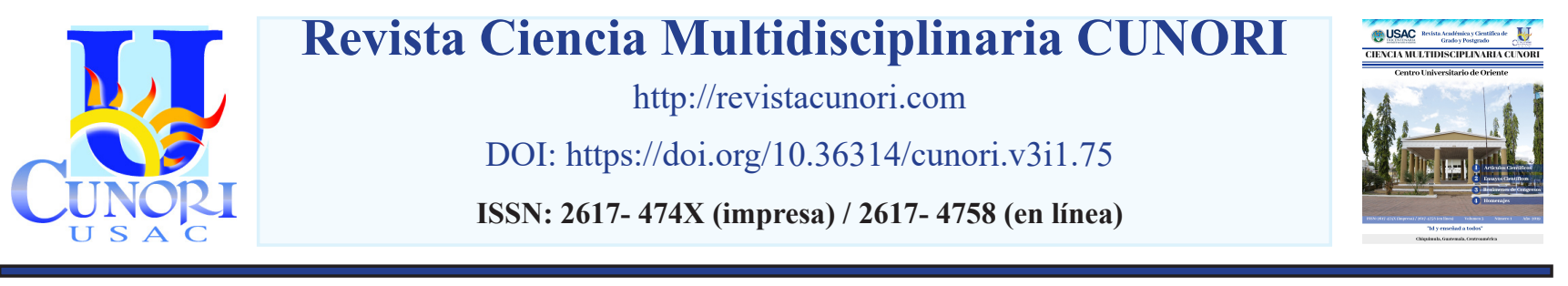

Como citar el artículo

Ramírez, N., Lemus, Y., Retana, R., Mazariegos, E., Arriola, C., (2019). Relación del monitoreo cardiotocográfico intraparto con el estado clínico del recién nacido. Revista Ciencia Multidisciplinaria CUNORI, 3(1), 11-17. DOI: https://doi.org/10.36314/cunori.v3i1.75

\title{
Relación del monitoreo cardiotocográfico intraparto con el estado clínico del re- cién nacido
}

\section{Relation of intrapartal cardiotocographic monitoring with the clinical status of the newborn}

\author{
Nancy Ramirez*, Yaneliz Lemus, Ronaldo Retana, Edvin Mazariegos, Carlos Arriola \\ Centro Universitario de Oriente (CUNORI), Universidad de San Carlos de Guatemala \\ Recibido: 24 de julio de 2018 / Revisión: 4 de septiembre de 2018 / Aceptado: 14 de enero de 2019 \\ Disponible en internet el 30 de agosto de 2019 \\ *Autor para correspondencia. \\ Correo electrónico: elizaramirezlopez69@gmail.com
}

Resumen

$\mathrm{E}$ 1 monitoreo fetal electrónico intraparto es la valoración del bienestar fetal basado en el registro de la respuesta cardíaca ante Los movimientos fetales y las contracciones uterinas, en la práctica obstétrica ayuda a predecir de manera acertada la condición fetal, la adaptación del medio intrauterino al extrauterino, es decir permite evaluar cuál sería el posible Apgar que obtendrá el recién nacido y de esta manera poder determinar la conducta obstétrica a seguir. Se realizó un estudio analítico transversal en el hospital nacional de Chiquimula, en el cual se evaluó la relación entre el monitoreo cardiotocografico fetal intraparto realizado a 271 pacientes con trabajo de parto activo y el estado clínico del recién nacido; a través de medidas de asociación se determinó que las pacientes con un monitoreo cardiotocografico fetal intraparto positivo tendrán 9.2 veces más riesgo de tener un recién nacido con un estado clínico desfavorable que aquellas con un monitoreo cardiotocografico fetal intraparto negativo ( $\mathrm{RR}=9.2 ; 2.722-31.08$ IC 95\%), de igual manera se determinó que el monitoreo cardiotocografico fetal intraparto positivo tiene una capacidad moderada para predecir un recién nacido con estado clínico desfavorable ( Sensibilidad $60 \%$; VPP $=16 \%$ ) pero el monitoreo cardiotocografico fetal intraparto es muy bueno como predictor del bienestar fetal ( Especificidad 88\%; VPN=98\%) mediante el test exacto de Fisher se obtuvo que el valor P, para dicha relación fue de $0.0007193(<0.05)$. Se concluyó que el monitoreo cardiotocográfico intraparto tiene relación con el estado clínico del recién nacido.

Palabras clave: monitoreo cardiotocográfico intraparto, estado clínico del recién nacido, apgar

\section{Abstract}

$\mathrm{T}$ he electronic intrapartum fetal monitoring is a method that serves for the assessment of fetal well-being based on the observation of the cardiac response to fetal movements and uterine contractions, in obstetric practice it helps to predict correctly the fetal condition, the adaptation from intrauterine to extrauterine environment, it allows us to evaluate what would be the possible Apgar that the newborn will obtain at the moment of birth and in this way to be able to determine the obstetric behavior to follow. A cross-sectional analytical study was conducted on intrapartum cardiotocography monitoring in the national hospital of Chiquimula to 271 patients with active labor, later the clinical status of the newborn was evaluated and it was related to intrapartum cardiotocography monitoring, it was obtained that the relative risk for this ratio was $9.2(2,722-31.08,95 \% \mathrm{CI})$. 
The intra-partum cardiotocographic monitoring performed on the labor and delivery service patients of the national hospital of Chiquimula had a sensitivity of $60 \%$ and a specificity of $88 \%$, the capacity of the non-reactive cardiotocographic monitoring to predict a clinical state of the unfavorable newborn is of $16 \%$, and the capacity of reactive cardiotocographic monitoring to predict a favorable clinical status of the newborn is $98 \%$. Through Fisher's exact test, the $\mathrm{P}$ value was obtained, for this ratio it was $0.0007193(<0.05)$. It was concluded that intrapartum cardiotocographic monitoring is related to the clinical status of the newborn.

Keywords: intrapartum cardiotocographic monitoring, clinical status of the newborn, apgar

\section{Introducción}

Los principios de la vigilancia electrónica del feto fueron publicados por primera vez en los últimos años de la década de 1,960 Hammacher, Hon, Caldeyro-Barcia y poco después se dispuso de los monitores comerciales. Hoy en día, la vigilancia electrónica fetal se utiliza ampliamente en todo el mundo, predice la ausencia de asfixia con mayor precisión que cualquier otra técnica o combinación conocidas. Proporciona información sobre el mecanismo de la asfixia y la posibilidad de mejorar estos problemas con el tratamiento conservador, mejora el resultado fetal perinatal, en especial al reducir el riesgo de óbitos intraparto y puntuación baja de Apgar (Navarro, 2006).

Según la Organización Mundial de la Salud en lo que respecta a muertes fetales se calculan 2.6 millones en todo el mundo, de los cuales el $71 \%$ se producen durante el trabajo de parto asociado a complicaciones obstétricas no tratadas oportunamente. (Organización Mundial de la Salud. 2017) En Latinoamérica la mortalidad neonatal es el indicador que más contribuye a la mortalidad en la niñez. Se calcula que la tasa de mortalidad neonatal es de 15 por cada 1,000 nacidos vivos, representando el $60 \%$. Las tasas más altas de mortalidad neonatal se encuentran en Haití, Bolivia y Guatemala (Organización Panamericana de la Salud, 2014).

En Guatemala la tasa de mortalidad perinatal es de 31 por 1,000 nacidos vivos, de la cual los mortinatos constituyen el $60 \%$ de esta cifra, la tasa de mortalidad neonatal es de 17 por 1,000 nacidos vivos, de esta, la neonatal temprana representa el $71 \%$. El objetivo de la medicina perinatal y de la vigilancia intraparto, es disminuir las tasas de morbimortalidad fetal y materna. Para ello se estudia al feto durante el proceso de embarazo y parto; en un intento de seleccionar aquellos fetos que se encuentran en situación comprometida o riesgo de hipoxia, con la intención de corregirla o evitarla antes que se produzcan efectos irreversibles (Navarro 2006).

El monitoreo cardiotocográfico intraparto se encarga de proporcionar un registro continuo de frecuencia cardíaca fetal, actividad uterina y movimientos fetales, es un método de fácil realización e interpretación, no invasivo, de bajo costo, reproducible, sin efectos secundarios ni contraindicaciones, en la actualidad para el control de la frecuencia cardíaca fetal comúnmente se utiliza la monitorización electrónica cardiotocográfica continua o intermitente, y el uso de la auscultación con fonendoscopio en los embarazos de bajo riesgo (Salcedo et. al 2015). 
El fin de vigilar la actividad cardíaca fetal, es identificar precozmente alteraciones del bienestar fetal como producto de oxigenación inadecuada y reconocer tempranamente el estado fetal insatisfactorio o no tranquilizador, ello permite tomar decisiones clínicas en beneficio del feto, obteniendo recién nacidos en mejores condiciones clínicas y reducción en la morbimortalidad neonatal (Salcedo et. al 2015). La frecuencia cardíaca fetal es controlada por el sistema nervioso autónomo (simpático y parasimpático). Su normalidad depende de la indemnidad de estos sistemas, los cuales son sensibles a cambios de la oxigenación fetal (Valdez, 2003).

Cuando los intercambios feto-maternos se deterioran y el aporte de oxigeno es inferior al necesario, el feto desencadena una serie de mecanismos que tienden a utilizar el oxígeno de reserva y a metabolizar la sustancia energética por excelencia (glucosa) por la vía anaeróbica con el fin de mantener las necesidades vitales, comportando la acumulación de metabolitos ácidos, que en un primer momento serán eliminados gracias a los mecanismos de compensación. A medidas que los intercambios empeora, la situación fetal se agrava y por consiguiente hay pérdida de bienestar fetal (Nava \& Zúñiga 2009).

La relación entre los movimientos fetales y las aceleraciones de la frecuencia cardíaca fetal, a medida que avanza la gestación, va haciéndose cada vez más estrecha hasta llegar al punto que al término de la gestación es un parámetro confiable para valorar el bienestar y la madurez fetal (Nava \& Zúñiga 2009).

\section{Material y métodos}

De los meses de abril a mayo del presente año se identificaron un total de 271 pacientes con trabajo de parto, atendidas en el servicio de labor y partos del hospital nacional de Chiquimula, los cuales cumplieron con los criterios de inclusión y exclusión; se procedió al estudio de los pacientes llenando una historia clínica y realizándose el monitoreo cardiotocográfico fetal intraparto durante 20 minutos, se interpretaron como un trazo reactivo y no reactivo, posteriormente se evaluó el estado clínico del recién nacido mediante la puntuación Apgar y características del líquido amniótico. Se ordenaron los datos

en tablas de contingencia correlacionando los resultados del monitoreo cardiotocográfico con el estado clínico del recién nacido. 


\section{Resultados}

\section{Relación del monitoreo cardiotocográfico intraparto con el estado clínico del recién nacido}

De 271 pacientes a quienes se les realizó el monitoreo cardiotocográfico intraparto, 233 (85\%) pacientes presentaron un trazo reactivo, de los cuales 4 (1\%) fueron recién nacidos con un estado clínico desfavorable; 38 (12\%) pacientes obtuvieron trazos no reactivos de los cuales $6(2 \%)$ recién nacidos presentaron un estado clínico desfavorable.

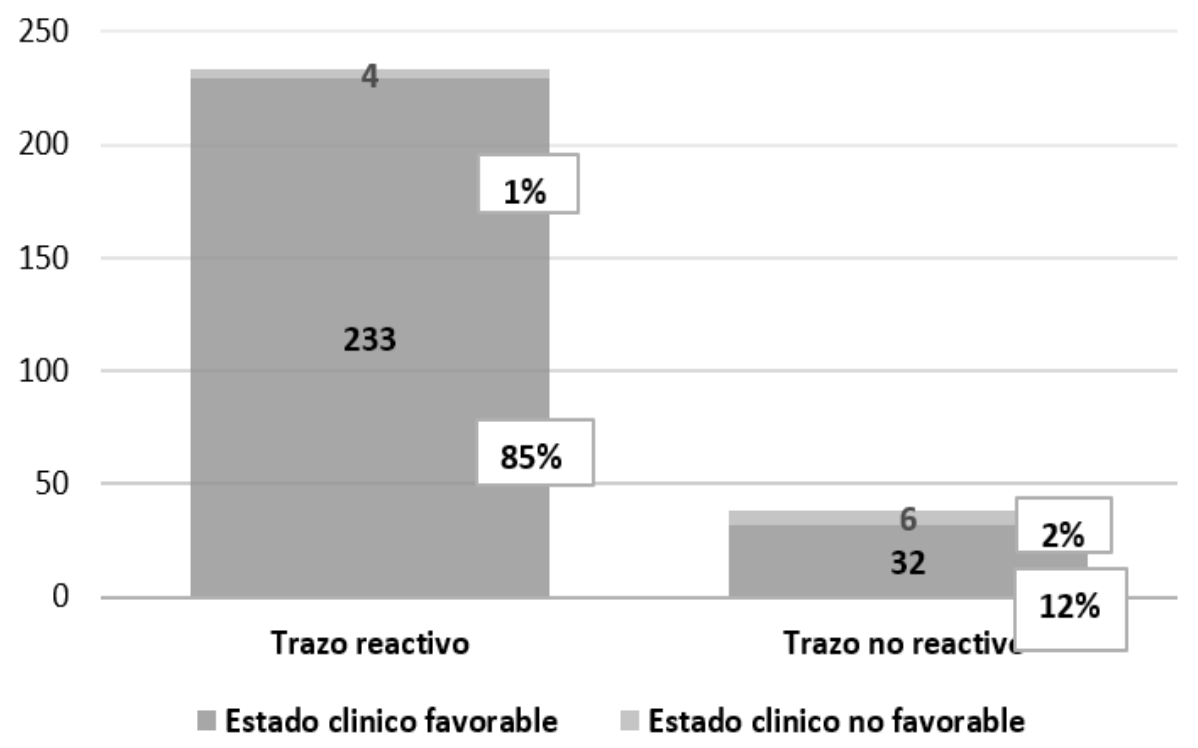

Figura 1. Relación del monitoreo cardiotocográfico intraparto con el estado clínico del recién nacido en pacientes ingresadas en el área de labor y partos del Hospital Nacional Carlos Manuel Arana Osorio de Chiquimula.

Las pacientes con un trazo no reactivo intraparto tendrán el riego de tener un recién nacido con un estado clínico desfavorable de 9 veces más que aquellas pacientes con un trazo reactivo, dichos resultados se correlacionan a un valor $\mathrm{P}<0.05$ rechazándose la hipótesis nula.

$\mathrm{RR}=9.2(2.722-31.08 ; \mathrm{IC} 95 \%)$

$\mathrm{P}=0.0007193$

Tabla 1. Determinar la relación del monitoreo cardiotocográfico intraparto con el estado clínico del recién nacido, en pacientes del servicio de ginecología y obstetricia del Hospital Nacional de Chiquimula Carlos Manuel Arana Osorio. 
Sensibilidad y Especificidad del monitoreo cardiotocográfico intraparto en relación al estado Clínico del recién nacido

El monitoreo cardiotocográfico intraparto demostró tener una sensibilidad de $60 \%$ y una especificidad del $88 \%$.

\begin{tabular}{|l|l|l|l|}
\hline & Positivo & Negativo & Total \\
\hline Positivo & $6(\mathrm{a})$ & $32(\mathrm{~b})$ & 38 \\
\hline Negativo & $4(\mathrm{c})$ & $229(\mathrm{~d})$ & 233 \\
\hline Total & 10 & 261 & $\mathbf{2 7 1}$ \\
\hline
\end{tabular}

Sensibilidad: $(\mathrm{a} / \mathrm{TP} \times 100 \%)=6 / 10 \times 100 \%=60 \%$

\begin{tabular}{|l|l|l|l|}
\hline & Positivo & Negativo & Total \\
\hline Positivo & $6(a)$ & $32(\mathrm{~b})$ & 38 \\
\hline Negativo & $4(\mathrm{c})$ & $229(\mathrm{~d})$ & 233 \\
\hline Total & 10 & 261 & $\mathbf{2 7 1}$ \\
\hline
\end{tabular}

Especificidad: $(\mathrm{d} / \mathrm{TN} \times 100 \%)=229 / 261 \times 100 \%=88 \%$

Cuadro 1. Sensibilidad y especificidad del monitoreo cardiotocográfico intraparto en relación al estado clínico del recién nacido, de las pacientes ingresadas al servicio de labor y partos del departamento de ginecología y obstetricia del Hospital Nacional Carlos Manuel Arana Osorio de Chiquimula.

Valor predictivo positivo y valor predictivo negativo del monitoreo cardiotocográfico intraparto en relación con el estado clínico del recién nacido

La presente tabla demuestra que el monitoreo cardiotocografico intraparto no reactivo demostró un valor predictivo positivo de $16 \%$ y un valor predictivo negativo de $98 \%$ en relación al estado clínico del recién nacido.

Valor predictivo positivo: $(\mathrm{a} / \mathrm{TP} \times 100 \%)=6 / 38 \times 100 \%=16 \%$

Valor predictivo negativo: $(\mathrm{d} / \mathrm{TN} \times 100 \%)=229 / 233 \times 100 \%=98 \%$

Cuadro 2. Valor predictivo positivo y valor predictivo negativo del monitoreo cardiotocográfico intraparto en relación con el estado clínico del recién nacido, de las pacientes ingresadas al servicio de labor y partos del departamento de ginecología y obstetricia del Hospital Nacional Carlos Manuel Arana Osorio de Chiquimula. 


\section{Discusión}

Se determinó que las pacientes con un monitoreo cardiotocografico fetal intraparto positivo tendrán 9.2 veces más riesgo de tener un recién nacido con un estado clínico desfavorable que aquellas con un monitoreo cardiotocografico fetal intraparto negativo ( $R R=9.2 ; 2.722-31.08$ IC 95\%), igualmente se determinó que el monitoreo cardiotocografico fetal intraparto positivo tiene una capacidad moderada para predecir un recién nacido con estado clínico desfavorable ( Sensibilidad 60\%; VPP $=16 \%$ ) pero el monitoreo cardiotocografico fetal intraparto es muy bueno como predictor del bienestar fetal ( Especificidad $88 \%$; VPN= 98\%) mediante el test exacto de Fisher se obtuvo que el valor P, para dicha relación fue de $0.0007193(<0.05)$. Se concluyó que el monitoreo cardiotocográfico intraparto tiene relación con el estado clínico del recién nacido.

\section{Agradecimientos}

Al Hospital Nacional de Chiquimula, en especial al comité de Docencia e Investigación por la aprobación para realizar la investigación. Un agradecimiento especial al Dr. Ronaldo Retana por su apoyo durante la investigación.

\section{Referencias bibliográficas}

Organización Mundial de la Salud -OMS. (2017). Reducir la mortalidad de los recién nacidos (en línea, sitio web). Ginebra, Suiza. (Notas descriptivas). Consultado 5 feb. 2018. Disponible en http:// www.who.int/mediacentre/factsheets/fs333/es/

Organización Panamericana de la Salud, Estados Unidos -OPS; Organización Mundial de la Salud -OMS, Suiza. (2014). Países de América Latina y el Caribe registraron avances en la reducción de la mortalidad materna, según nuevo informe de la ONU (en línea, sitio web). Washington, Estados Unidos. Consultado 7 feb. 2018. Disponible en http://www.paho.org/uru/index.php?option=com_content\&view=article\&id=839:once-paises-de-america-latina-y-el-caribe-registraron-avances-en-la-reduccion-de-la-mortalidad-materna-segun-nuevo-informe-de-la-onu\&Itemid=340

Salcedo, F; Méndez, R. \& Vallejo C. (2015). Vigilancia fetal durante el trabajo de parto (en línea). Revista Ciencias Biomédicas 6(1): 170-178. Consultado 5 feb. 2018. Disponible en www.revista.cartagenamorros.com/pdf/6-1/23_guia_vigilancia.pdf

Nava, E. \& Zuñiga D. (2009). Electrocardiotocografia intraparto (en línea). Acta Medica Grupo Ángeles 7(1):24-28. Consultado 5 feb. 2018. Disponible en http://www.medigraphic.com/pdfs/actmed/am2009/am091d.pdf

Navarro, M. 2006. Relación entre las desaceleraciones de la frecuencia cardíaca fetal y valores de pulsioximetria fetal. Universidad de Granada. 321 p. Consultado 4 feb. 2018. Disponible en https:// hera.ugr.es/tesisugr/15923587.PDF

Valdez, E. (2003). Rol de la monitorización electrónica fetal intraparto en el diagnóstico de sufrimiento fetal agudo (en línea). 5 ed. Chile, SOCHOG p.411-419. Consultado 6 feb. 2018. Disponible en https://scielo.conicyt.cl/scielo.php?pid=s0717-75262003000500010\&script=sci_arttext. https://doi. org/10.4067/S0717-75262003000500010 


\section{Sobre la autora}

\section{Nancy Elizabeth Ramírez López}

Médico y Cirujano, egresada del Centro Universitario de Oriente CUNORI de la Universidad de San Carlos de Guatemala en el año 2018. Realizó la investigación que lleva como título "Relación del Monitoreo Cardiotocográfico intraparto con el estado clínico del recién nacido". Actualmente se desempeña como médico residente 1 de Ginecología y Obstetricia en el Hospital Regional de Zacapa.

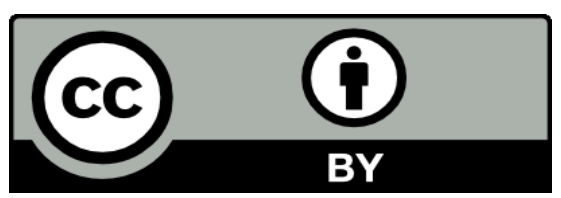

Este texto está protegido por una licencia CreativeCommons 4.0.

Usted es libre para compartir, copiar y redistribuir el material en cualquier medio o formato y adaptar el documento, remezclar, transformar y crear a partir del material para cualquier propósito, incluso comercialmente, siempre que cumpla la condición de atribución: usted debe reconocer el crédito de una obra de manera adecuada, proporcionar un enlace a la licencia, e indicar si se han realizado cambios. Puede hacerlo en cualquier forma razonable, pero no de forma tal que sugiera que tiene el apoyo del licenciante o lo recibe por el uso que hace. 